\title{
Interaction of Methylphenidate and External Cue in an ADHD Behavioral Training Model
}

\author{
Erica Kimmick $^{\mathrm{a}}$, Robin McGovern ${ }^{\mathrm{a}}$, and Alan Gittis ${ }^{\mathrm{a}}$
}

Symptoms of Attention Deficit Hyperactive Disorder (ADHD) include hyperactivity, inattentiveness, and impulsivity, which are caused by dopamine dysfunction in the medial prefrontal cortex. A six-hydroxydopamine lesion model of ADHD was used to examine response accuracy on an attentional task under one of three conditions: cue only, methylphenidate only (MPH), and MPH with cue. Results indicated rats injected with methylphenidate and exposed to an external cue showed a significant increase in response accuracy compared to the MPH and cue only groups. Also, the results indicate animals in the cue only condition had an increase performance in response accuracy compared to just methylphenidate group. This study provides evidence that combination of a stimulant and external cue would have a stronger affect than a stimulant or external cue was given alone.

Keywords: dopamine, hyperactivity, methylphenidate, external cue, medial prefrontal cortex

\section{Introduction}

Attention Deficit Hyperactivity Disorder (ADHD) has become the most frequently diagnosed $(6.8 \%)$ behavioral disorder among school-age children (Furman, 2009) with symptoms including hyperactivity, inattentiveness, and impulsivity (Barkley, 2003). According to Bloom, Cohen, and Freeman (2010), out of five million children aged 3-17 years, $11 \%$ of boys were about twice as likely as girls $(6 \%)$ to have ADHD. Additionally, in recent years there has been an increase in the number of adults diagnosed with ADHD. With the increasing amounts of patients being diagnosed with ADHD, the number of prescriptions for ADHD medication has also increased. As such, to treat ADHD symptoms, stimulant medications (Zachor, Roberts, Hodgens, Isaacs, \& Merrick, 2006) like Dexedrine and Adderall (American Academy of Pediatrics, 2001; Spencer, Biederman, \& Wilens, 2000) are prescribed; however, Ritalin (Methylphenidate), a stimulant medication, is more widely prescribe to treat ADHD symptoms (Pelham et al., 1999).

Furthermore, there is much needed attention to evaluate the effectiveness of not only ADHD medication, but also behavioral therapy treatment. Wilens (2008) found that in human trials, ADHD symptoms were reduced by the presence of methylphenidate and amphetamines. However, other studies, using human subjects, have reported success in treating symptoms of ADHD with non-pharmacological (Toplak, Conners, Schuster, Knezevic, \& Parks, 2008). For example, Klingber, Forssberg, \& Westerberg (2002) used a computer-based working memory training sequence to see if working memory performance would improve symptoms of ADHD. The results showed working memory performance improved and in a follow up study there was improvement in response inhibition and inattentiveness (Klingber et al., 2005). Since there is evidence that pharmacological and nonpharmacological treatment alone decreases ADHD symptoms; combining these treatments may have a greater affect improving ADHD symptoms.

The current study investigated the interaction of behavioral training and the enhancement of dopaminergic activity in the medial prefrontal cortex (mPFC) that acted synergistically to improve behavioral inhibition in an animal model of hyperactivity. More specifically, the current study focused on the medial prefrontal cortex (mPFC) and the effects of a stimulant, methylphenidate (MPH) and an external cue (light) for response inhibition.

\section{Dopamine (DA)}

Dopamine is a major catecholamine neurotransmitter, produced in the Ventral Tegmental area (VTA) and plays a role in reward system, motivation, attention, working memory, and learning (Carboni, Silvagni, Valentini, \& Chiara, 2003). Dopaminergic neurons are located in the midbrain, specifically, VTA and project to three dopamine pathways: the mesolimbic, nigrostriatal, and mesocortical. The mesolimbic dopamine neurons of the VTA project to the limbic system, which plays a key role in mediating reward, and has been implemented in addiction (Cao et al., 2010). The current study focused on the mesocortical DA pathway, which initiates from the VTA and projects to the mPFC, where it plays a role in reward system, motivation, attention, and working memory (Carboni et al., 2003), which relates to ADHD. Bidwell, McClernon, \& Kollins (2011) explained the etiology symptoms of ADHD resulting from decrease dopamine activity in the mesocortical pathway, which leads to attentional deficits. DA neurons are associated with the brain's ability to predict errors in behavior and maximize the rewards for a suitable behavior. With the loss of DA neurons there will be a dysfunction in behavior, attention, and rewards.

\section{Medial Prefrontal Cortex}

In the past, studies focused on brain regions like the striatum and nucleus accumbens to treat ADHD symptoms (Almaric \& Koob, 1993; Casey, B. J., 1997; Carboni et al., 2003); however studies are now focusing on the association of hyperactivity and inattentiveness involved within the medial Prefrontal Cortex (mPFC) (Fuster, 1997; Koda, 2010; Wanchoo, 2010). 
Repeated notions have been produced that there are similarities between symptoms of ADHD and lesions or injuries to the mPFC (Barkley, 1997; Benton, 1991; Mattes, 1980). Prefrontal region injuries demonstrate deficits in sustained attention, inhibition, regulation of emotion and motivation, and the capacity to organize behavior across time (Fuster, 1997; Grattan \& Eslinger, 1991; Stuss \& Benson, 1986). Positron Emmision Tomography (PET), Coaxial Tomographic (CT), and Magnetic Resonance Imaging (MRI) are just a few tools that have been used to identify neuroanatomical correlates of ADHD. Studies using PET to assess cerebral glucose metabolism have found diminished activity in the mPFC in adults with ADHD (Schweitzer \& Sulzer-Azaroff, 1995; Zametkin et al., 1990). This finding suggests that a smaller mPFC or lack of frontal asymmetry (a smaller than normal right frontal region) probably mediates the expression of ADHD symptoms (Castellanos, 1996 \& Barkley, 1997).

The medial prefrontal cortex ( $\mathrm{mPFC}$ ) manages behavior processes, decision-making, and moderates social behavior. It is theorized that ADHD symptoms are caused by a deficit in executive functions (Pennington \& Ozonoff, 1996) and that several neural circuits are involved, including the medial prefrontal cortex (Casey et al., 2002; Pennignton, 2002). The executive system may have evolved to support the social activities of reciprocal exchange and altruism, imitation and vicarious learning, self-sufficiency and innovation, and social self-defense (Barkley, 2003). Executive function establishes the ability to differentiate conflicting thoughts, clarify good from bad, and suppress one's urges that may ultimately be unacceptable. In an ADHD patient, his or her executive function is the cause of poor inhibition of behavioral responses, or what Nigg (2001) has called "executive inhibition," which is established as impaired in this disorder (Barkley, 2003). Executive inhibition associates individual problems with nonverbal and verbal working memory, planning, responding perseveration, motor sequencing of time, and other frontal lobe functions (Barkley, 2003). Thus, executive inhibition played an important role in this study because the presence of methylphenidate, in the mPFC, and the external cue increased the rats' behavior performances, which led to improve executive function.

\section{Methylphenidate}

Methylphenidate (MPH), also known as Ritalin, is a stimulant medication prescribed for the treatment of ADHD and is highly effective in the treatment of its symptoms in both children and adults (Spencer et al. 1996; Taylor \& Russo 2001). Wilens (2008) revealed that $70 \%$ of children, taking MPH, showed a reduction of their core symptoms of ADHD. Stimulant medications are used because of their ability to increase arousal or alertness. Given their structural similarity to certain brain neurotransmitters, they are considered sympathomimetic compounds (Dupaul \& Barkley, 1990). Therefore, stimulants are like agents that imitate the stimulation of the sympathetic nervous system. While dopamine is an important part of the sympathetic nervous system, the presence of MPH will block dopamine transporters (DAT) to increase extracellular DA (Volkow, Fowler, Wang, \& Goldstein, 2002). MPH does so through the reuptake inhibition of the monoamine transporters, which increases extracellular levels of DA in the mPFC (Koda, 2010).

\section{Drug Administration}

There has been much controversy over prescribing acute versus chronic administration of MPH. The consequence of acute administration of MPH is that it does not suppress the hyperactivity and impulsivity all day because of the drugs half-life. Meaning, MPH is only in the system for six hours, so if MPH was chronically administered the symptoms would most likely be suppressed all day (Barkley, 2003). Koda (2010) explored the difference between acute versus chronic administration of MPH in a rat model. He concluded that even though acute and chronic administration of MPH increased extracellular DA levels in the prefrontal cortex, chronic administration lasted longer than acute administration. Overall, the current study strengthened the idea that chronic administration with a consistent dose of MPH $(3 \mathrm{mg} / \mathrm{kg})$ played a pivotal role in the improvement of behavioral performance.

\section{6-Hydroxydopamine}

Neurotoxin 6-hydroxydopamine (6-OHDA) selectively destroys catecholaminergic neurons (Iancu, Mohapel, Brundin, \& Paul, 2005). Meaning, 6-OHDA kills dopaminergic neurons through dopamine reuptake transporters, which causes hyperactivity in rats (Shaywitz et al., 1976). The behavior of these animals is said to be very similar to that of children with ADHD and includes hyperactivity, and learning deficits (Shaywitz \& Shaywitz, 1989). This study used dopamine depletion via 6-OHDA lesions of the medial pre-frontal cortex to model ADHD, and to counteract the lesions, MPH was used to chemically remediate the deficit.

\section{DRL 30s}

To evaluate the interaction of a pharmacological and behavioral control technique, this study employed a differential reinforcement of low rate (DRL 30s) task. It has been shown that animals with prefrontal DA depletions were impaired on the DRL task (Sokolowski, 1994). DRL 30 tests for impulsivity and inhibitory control (Neill, 1976 \& Bull, Reavill, Hagan, Overend, \& Jones, 2000). That is known to be dependent on DA function with the mPFC (Sokolowski \& Salamone, 1994). The task evaluated performances of animals suffering from mPFC lesions and how their performances were affected by a time delay.

This technique was effective for behavioral inhibition because it trained the rat to respond to a stimulus (food pellet) with a positive reinforcement rather than a negative reinforcement. Before the rat received the pellet it needed to wait for the allotted time (i.e. 30 seconds) to pass and then it was able to press the lever to receive its pellet. If the rat pressed the lever before the allotted time (30 seconds) they did not receive their pellet. The timer would reset and the rat would have to wait until the time delay was over. This process was continuous throughout the experiment. Since the rats lost interest during the 30-second delay sessions, they were tested with a 20 second delay for the experiment. 


\section{Methylphenidate and behavioral treatment}

Uncontrolled cohort studies have suggested that multimodal interventions combining behavioral and pharmacological treatments might reduce ADHD symptoms (Satterfield et al., 1981). Pelham et al. (1988) found that a combination of methylphenidate and classroom behavior therapy yielded great reductions in teacher ratings of inattentive overactive behavior than behavior therapy alone. Similarly, Richardson et al. (1988) reported that children with ADHD that responded favorably to MPH had greater gains in reading grade equivalents during a remedial program than children with ADHD who responded less favorable to MPH (Sandberg, 1996). Combining behavior therapy and medication treatment could permit lowering the dosage of the medication. In addition, Hinshaw et al. (1984) found that MPH increased the accuracy of his/her ADHD self-evaluation in a cognitive-behavioral intervention. Even more studies have confirmed that behavior and medication treatment are related and could ultimately improve hyperactivity to where less and less of the drug is needed (Barkley, 2003; Koda, 2010).

\section{Light}

The house light establishes an external cue for behavioral model purposes in the operant chamber. The presence of light was a non-biological cue that was used as a behavioral treatment to try and increase their accuracy on behavioral performance. Studies have shown that a behavioral cue reduces the hyperactivity/impulsivity symptoms of ADHD. The current study used the house light that signaled when not to press the lever. If the rats pressed the lever while the light was on a food pellet was not dispensed. Instead, the timer reset and the rats waited until the time delay was over and the light was off to press the lever again and receive a food pellet.

This study modeled the effects of MPH and behavioral control techniques in a rat model of hyperactivity. The aim of this study was to establish the effect of chronic MPH with or without cue on behavioral performance on an operant task. It is hypothesized rats given MPH and exposed to the external cue would have a better behavioral performance than rats given a cue or MPH alone. The external cue was important, because it allowed the rats to have a visual cue, rather than a biological cue, to decide whether or not to press the lever.

\section{Method}

\section{Design}

The design of the study was a 2 × 2 factorial design. Rats were randomly assigned into three groups: lesion with methylphenidate and cue $(\mathrm{M}+\mathrm{L})$; lesion with just methylphenidate $(\mathrm{M})$; and lesion with just cue (L). Each group was tested in the operant chamber measuring the rats' behavioral performances.

\section{Animals}

Eight adult male Long Evans Hooded Rats served as subjects. Each subject completed a baseline test and experimental test in the DRL 30 operant chamber. They were housed individually and given free access to water (14.2-14.5 $\mathrm{g}$ of food after each session and animals were maintained at $85 \%$ of their body weight throughout the baseline and experimental testing.

\section{Materials/Apparatus}

\section{Behavioral procedures}

For this experiment, an operant paradigm (DRL 30) (Dimensions $33.5 \mathrm{~cm}$ by $32 \mathrm{~cm}$ ) was used to examine behavioral performance of the three conditions. Rats underwent two testing phases for this experiment: baseline and experimental testing on the DRL-30 schedule (differential reinforcement of low rates) and weighed before each session. At the beginning of their baseline testing the lever was unavailable and for 15 minutes the rats were given food pellets according to a variable interval scale on the Coulbourn instrument module, so they were not able to predict when the next pellet was dispensed. This occurred over the period of three sessions. After the three sessions, the lever was available and the rats were trained to press the lever for pellets. Also at this time, the house light was introduced. The baseline testing determined the rat's natural performance on the task without any surgery or MPH to interfere their natural judgment. Once the rats were habituated to the operant chamber, they started their baseline testing. It was crucial that in order for each rat to proceed to the next time delay schedule they had to reach a predetermined accuracy level (.300) (Peterson, Wolf, \& White, 2003). Many studies used the predetermined accuracy level, because it showed that the rats learned the operant task.

Their first time delay schedule was a five second delay, which took them two sessions to reach the predetermined accuracy level. Second time delay was a ten second delay that took them three sessions to learn. The third time delay was a 15 second delay that was learned in seven sessions. The fourth time delay was a 20 second delay for seven sessions. Finally, a time delay of 30 seconds was learned in eight sessions. The rats were exposed to each session for 30 minutes. Once each rat reached the predetermined accuracy level (.300) for each delay (DRL 5, 10, 15, 20, 30 seconds) they were ready for surgery. After surgery, the rats underwent their experimental testing, which was similar to their baseline testing.

\section{Procedure}

Dopamine (DA) depletion by medial prefrontal cortex lesion

The depletion of DA was produced by the infusion of 6OHDA (Research Biochemicals Inc.) while the rats were under anesthesia $(60 \mathrm{mg} / \mathrm{kg}$ sodium pentobarbital). The 6OHDA was injected in the medial prefrontal cortex at $3.2 \mathrm{~mm}$ anterior to Bregma, $0.6 \mathrm{~mm}$ lateral on each side of midline and $4.0 \mathrm{~mm}$ deep (Paxinos \& Watson Brain Atlas, 1986). There were two injections of $10 \mu \mathrm{l}$ of 6-OHDA solutions with $3 \%$ ascorbic acid. First, the needle was kept at $4.0 \mathrm{~mm}$ and $5 \mu \mathrm{l}$ was injected. The needle stayed in that position for five minutes and then the needle was raised $1 \mathrm{~mm}$, raising the tip to $3.0 \mathrm{~mm}$ and another $5 \mu \mathrm{l}$ was injected. This was completed bilaterally. The 6-OHDA solution was made by dissolving $3 \mathrm{mg}$ 6-OHDA, $2 \mathrm{mg}$ of ascorbic acid per $\mathrm{ml}$ of $0.9 \%$ isotonic saline. Three percent ascorbic acid was added to prevent rapid oxidation of 6-OHDA (Wanchoo, 2010). Injecting 6-OHDA produced a medial prefrontal cortex lesion. The rats' post surgical recovery period was five days. 


\section{Methylphenidate (MPH)}

The current study was supplied with MPH by Westminster College. Methylphenidate was dissolved in $0.9 \%$ isotonic saline (Wanchoo, 2010), and administered through intraperitoneal injections (i.p.). Methylphenidate (M) and methylphenidate with light $(\mathrm{M} / \mathrm{L})$ conditions were weighed and injected with MPH ( $3 \mathrm{mg} / \mathrm{kg}$ dose $)$ at a volume of $.1 \mathrm{ml} / \mathrm{g}$ body weight before each testing.

\section{Light}

The cue was only given to rats in the cue only and methylphenidate and cue conditions during testing. The house light signaled when not to press the lever and if the lever was pressed when the light was on a food pellet was not dispensed. Instead, the timer reset and the rats waited until the light was off in order to press the lever again and receive a pellet.

\section{DRL30}

DRL 30 task was to increase the rate of probability of behavior performance by reinforcements. This was an effective technique for behavioral inhibition because it trained the rat to respond to the stimulus (food pellet) with a positive reinforcement rather than a negative reinforcement. Then before the rat could receive another pellet it waited for the allotted time (i.e. 30 seconds) to pass and then pressed the lever again and received another pellet. If the rat pressed the lever before the allotted time ( 30 seconds) they did not receive their pellet. Also, the timer reset and added more time so each rat would wait longer. This process was continuous throughout the experiment. Since the rats lost interest during the 30-second delay sessions, they were tested with a 20 second delay for the experiment.

After surgery rats were assigned to one of the four experimental groups, and trained for 30 minutes in the DRL 20 schedule for 25 days. Before each testing session rats were weighed and injected with either saline or MPH 15 minutes prior to testing. The Coulbourn Instrument module collected the lever press accuracy averages. The rats were tested for five days per week for five weeks. The DRL 20 task tested each rat's behavioral performance and once those accuracies were documented the author compared each rat's baseline testing and experimental testing to indicate whether their performances increased, decreased, or stayed the same.

\section{Statistical Analysis}

The mean lever press accuracy for total behavioral performance was determined after sessions of saline and methylphenidate administration. One-Way Anova post hocs and Least Significance Difference (LSD) tests were used to analyze the overall main effects of MPH and cue. A repeated measure analysis was conducted to assess if there was significance with each group's ability to learn the task in a specific amount of time. All statistical comparison maintained a significance level of 0.05 .

\section{Results}

A one-way between-groups analysis of variance was conducted to explore the overall main effects of methylphenidate (MPH) and cue. Participants were divided into four groups randomly (Control, $n=2$; Cue, $n=2 ; \mathrm{MPH}$, $\mathrm{n}=2 ; \mathrm{MPH}+\mathrm{Cue}, \mathrm{n}=2$ ). The averages were compiled in the one-way Anova test which revealed that there was a significant difference between control, cue, MPH, and MPH + Cue: $F(3,4)=7.371, p=.042, \eta_{p}{ }^{2}=5.48$. The Least Significance Difference (LSD) test indicated that the control $(\mathrm{M}=.324, \mathrm{SD}=.018)$ and $\mathrm{MPH}+\mathrm{Cue}(\mathrm{M}=.648, \mathrm{SD}=.047)$ were significantly different $(p=.015)$ from the MPH and Cue condition. In addition, the LSD test indicated that $\mathrm{MPH}(\mathrm{M}=$ $.336, \mathrm{SD}=.047)$ and $\mathrm{MPH}+\mathrm{Cue}(\mathrm{M}=.648, \mathrm{SD}=.047)$ were significantly different $(p=.017)$ from the Cue and Control condition. Therefore, the main effects of MPH + Cue had an overall better average compared to the other three conditions.

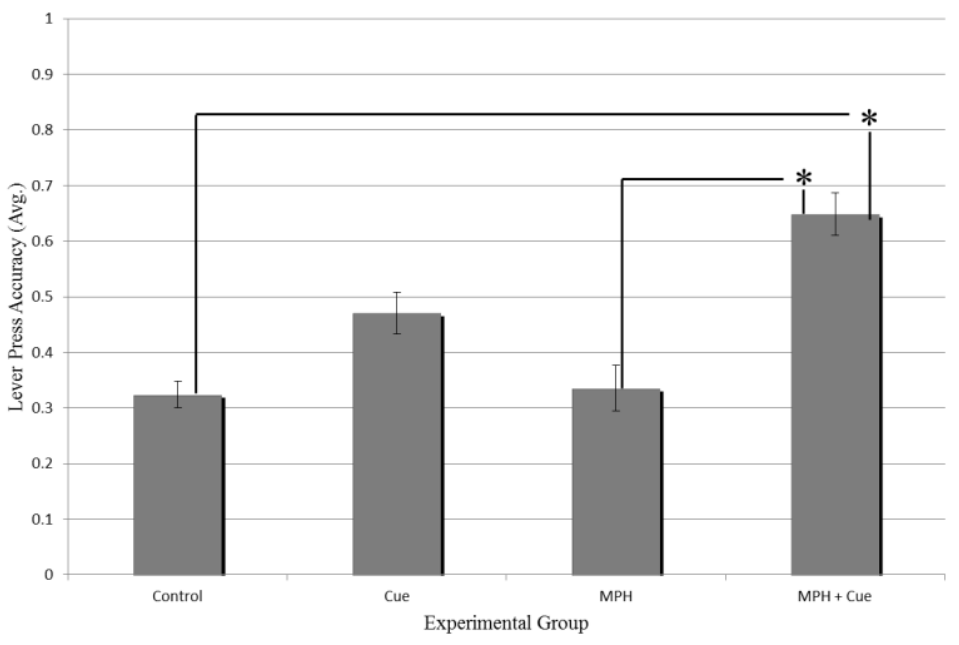

Figure 1: Mean difference values representing the total number of averages for each condition and overall main effects of methylphenidate and cue. Standard errors are represented in the figure by the error bars attached to each column. Control $(M=.342, S D=.018)$, JL $(M=.471$, $S D=.019), \mathrm{JM}(M=.337, S D=.148)$, and $\mathrm{M}+\mathrm{L}(M=.648$, $S D=.047)$. The asterisk means there is significance $(p<.05)$ between conditions. 
(a)

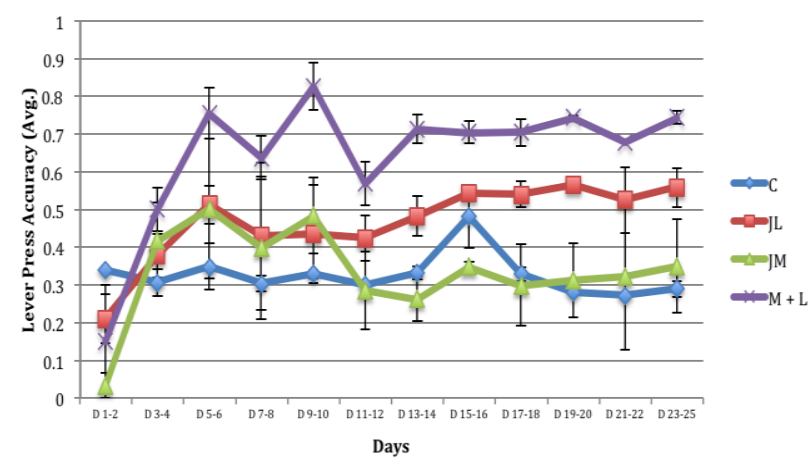

(b)

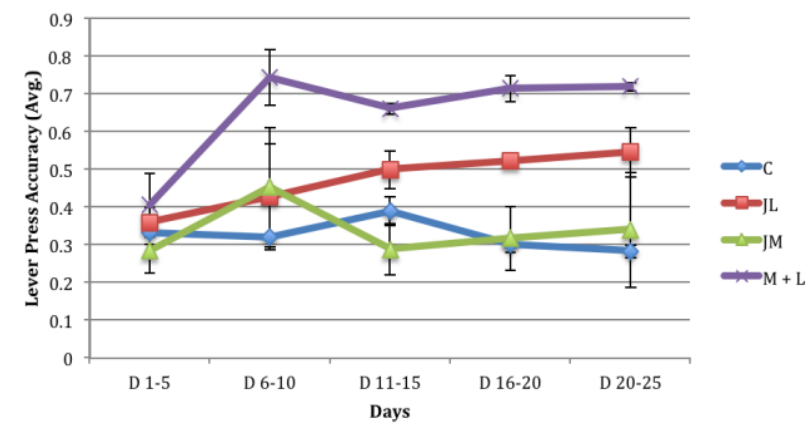

(c)

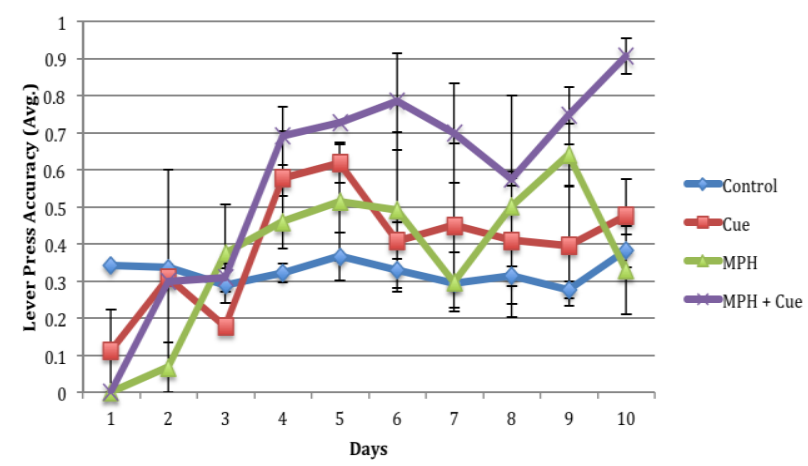

Figure 2: Effects of Methylphenidate $(3 \mathrm{mg} / \mathrm{kg}$ ) on behavioral performance on an operant task across 25 days of testing. (a) Averages of collapsed data in two-day bins. Behavioral performances stable in the first ten sessions. (b) Averages of collapsed data in five-day bins. (c) Averages through first ten sessions of testing. Standard errors are represented in the figures by the error bars attached to each point.

A mixed between-within subject's analysis of variance was conducted to determine each conditions measured acquisition of the task over a period of time (Figure 2a). The sessions were collapsed in two-day bins to view the data in a more compressed graph and to understand when the conditions acquired the task over time and how quickly the task was attained (i.e. Days 1 and 2 averages combined). There was significant main effect of session: $F(11,44)=$ $8.670, p<.01, \eta_{p}{ }^{2}=.684$. In addition, there was significance interaction between sessions*Cue $(F(11,44)=2.890, p=$ $\left..006, \eta_{p}{ }^{2}=.419\right)$ and sessions*MPH $(F(11,44)=3.044, p=$ $\left..004, \eta_{p}{ }^{2}=.432\right)$. There was no significant interaction of sessions*MPH*Cue $(M=.486, S D=.206)$. The betweensubjects effects test indicated that Cue is the only variable that had a significant effect on response accuracy $(F(1,4)=$ $\left.16.308, p=.016, \eta_{\mathrm{p}}{ }^{2}=.803\right)$. Therefore, the effect of sessions on Cue and MPH influenced the course of acquisition for each condition was significant, but the effect of session on the combination of Cue and MPH did not show significance on response accuracy.

Since there was significant effect of session when the data was analyzed in the two-day bins, the data was collapsed in a five-day bin graph (Figure 2b) to show the how the first ten sessions influenced the course of acquisition for each condition. Rats reached a stable level of performance within the first ten sessions, and after day ten the rat's performance was stable for the duration of the experiment. As such, the two-day bin and five-day bins data, from the first ten sessions, were compiled and examined the effects of drug and cue on the rat's acquisition of the task.

A mixed between-within subject's analysis of variance was conducted to assess the influence MPH and Cue on acquisition of the task (Figure 2c). There was significance with just sessions: $F(9,36)=10.071, p<.01, \eta_{p}{ }^{2}=.716$. In addition, there was significant interaction between sessions*Cue $\left(F(9,36)=2.613, p=.020, \eta_{p}{ }^{2}=.395\right)$ and sessions* MPH $(F$ 9, 36 $\left.)=3.550, p=.003, \eta_{p}{ }^{2}=.470\right)$ meaning cue and drug had significantly influence acquisition. There was no significant interaction between sessions*MPH*Cue $(M=.524, S D=.259)$ meaning the combination of drug and cue had no significant influence on acquisition. The between-subjects effects tests indicated there is no significance with the variables Cue, MPH, or Cue*MPH. Therefore, these results are similar to the two-day bins where the effect of sessions on Cue and MPH influenced the course of acquisition for each condition and had significance. However, the effect of sessions combined with Cue and MPH did not show significance.

\section{Discussion}

Behavioral performances combined with methylphenidate (MPH) and cue (light) resulted in significant increase in response accuracy on a DRL 20 operant task. This result confirmed the hypothesis that rat's injected with MPH and exposed to light $(\mathrm{M}+\mathrm{L})$ would have better acquisition performance on the DRL 20 task compared to MPH or cue alone. The $\mathrm{M}+\mathrm{L}$ condition was consistent throughout testing and had the highest lever press accuracy averages.

Unexpectedly, rats exposed to light revealed they had a better acquisition of behavioral performance on the DRL 20 schedule task than expected. Compared to the MPH condition, just light condition performed better on the DRL 20 task than the MPH condition. In addition, they showed stable response patterns after achieving the task. The light served as a visual cue to the rats and assisted them in their decision to press the lever.

It was also predicted that the rats injected with MPH would have improved performance on the DRL 20 task. In the beginning, they were learning the task well, and during days one thru four they had similar averages compared to $\mathrm{M}+$ L group. Days five thru ten the MPH condition accuracies started to decrease and were similar to the just cue accuracies. 
Then at day 11 their performances decreased even more and became similar to the control condition performances and stayed like this for the entire duration of the experimental testing. With subsequent testing days, MPH condition was unable to improve their accuracies to the level of the $\mathrm{M}+\mathrm{L}$ condition accuracies. It was hypothesized that $\mathrm{M}+\mathrm{L}$ and MPH condition would have similar accuracies, but from the condition's data they ended with dissimilar accuracies, which was unexpected. One reason for the inconsistency on acquiring the task may have been that the rats were not given enough MPH have similar performances as the $\mathrm{M}+\mathrm{L}$ condition. Since the rats were only exposed to MPH, they were at a disadvantage compared to the $\mathrm{M}+\mathrm{L}$ condition. It is thought that MPH condition may have need more MPH to reach the same performance levels as $\mathrm{M}+\mathrm{L}$ condition. In conclusion, the data for the MPH condition provided evidence that drug treatment decreased ADHD symptoms, but not significantly compared to the cue condition and $\mathrm{M}+\mathrm{L}$ condition.

Acquisition of the task was analyzed between all conditions. Sessions were significant in both two-day bins and the first ten session. Sessions were significant because they demonstrated the progress of each condition. The beginning sessions each condition had an up and down performance pattern and it was not until day 13 when each conditions performance pattern became stable. Interestingly, the learning curve in the two-day and five-day bins indicated similar significances, which suggested treatment of the conditions effected the learning curve. However, the learning curve, in either graphs, had no significant affect on the combination treatment of cue and MPH. This might be due because there was such a small number of animals.

Interestingly, the first ten sessions for each condition had an unstable pattern of increase and decrease performances. The reason for the instability with each conditions performances could have been influenced by the two weeks the rats were off due to their surgeries and recovery periods. Even though the rats learned the task during the baseline test, it did not take them as long to relearn the task and perform well, because with the addition of MPH, cue, or both increased their performances much faster.

When comparing the present findings with other researchers, it is hard to decipher if they were dissimilar, because there are very few if any present rat model studies that combined a pharmacological compound with a behavior treatment. Klingberg et al. (2005) attributed behavior treatment alone-reduced ADHD symptoms. On the other hand, Koda (2010) \& Wilens (2008) suggested MPH alone reduced ADHD symptoms and improved performance on the DRL 30 task. These results are similar to the present study that drug alone and behavior treatment alone do increase performance, but by combining the two treatments increased performance even more, which is displayed in the present study.

The strength of this study provided evidence that combining MPH and cue resulted in an increase performance on an operant task that exceeded the performances of MPHalone or cue-alone. This study also found that when rats treated with MPH or cue alone their performances on the operant task increased also, these results are similar with previous experiments (Klingber, 2005; Wilens, 2008; Koda, 2010). To strengthen this study, more testing days and test subjects would be beneficial to increase the data. Finally, performing histology on brain tissue would be beneficial to confirm that the 6-OHDA was properly administered in the mPFC.

Additionally, animals could be randomly assigned and tested in two periods. For example, the first period would have four rats in a condition (e.g., $\mathrm{M}+\mathrm{L}$ ) and tested for 30 days. When the 30 days are up that same condition would be tested for another 30 days, but under the a different condition (e.g., MPH). Finally, animals like Spontaneously Hypertensive Rat (SHR), Wistar-Kyoto (WKY), or SpragueDawley (SD) that are bred to have ADHD symptoms are beneficial, because no surgeries would be needed. In addition, using different tests like the water-maze test or the temporal response differentiation (TRD), or progressive ratio (PR) (Ferguson, 2007) would be beneficial. Ferguson (2007) found that the TRD performance proved to be less sensitive with MPH treatment than the DRL 30 performance, meaning there are less incorrect responses in the TRD performance than the DRL 30 performance.

In conclusion, this experiment provides evidence that combining a pharmacological and behavioral treatment treated the symptoms of ADHD better than a drug or cue alone. The clinical relevance to this study is the specific treatment for ADHD. There is much debate over whether to treat ADHD with stimulants or with behavioral treatments. According to Sonuga-Barke, Thompson, Abikoff, Klein, \& Brotman (2006), behavioral treatments work just as well as a drug treatment. For example, using a standard psychosocial approach (SPA) allows the child to interact with their parents and learn acceptable social behaviors, but it does not target the biological dysfunctions underlying ADHD. Another nonpharmacological intervention is called a New Forest Parenting Package (NFPP), which deals with a child-parent interaction of behavioral management and creating more positive interactions (Sonuga-Barke et al., 2006). Again it is found that this technique reduces the child's inattentiveness, but this technique needs to start at preschool age. On the other hand, pharmacological interventions resulted in decrease symptoms of ADHD and increase in performance. Present studies debate over acute versus chronic administration of MPH to decrease ADHD symptoms (Sprague \& Sleator, 1976; Richardson et al., 1988; Pelham et al., 1999; Barkley, 2003; Koda, 2010). They all confirm that chronic administration provides better performance and decrease symptoms of ADHD than acute administration. Although, Pelham et al., (1998) revealed in a classroom setting that when MPH and a classroom behavior therapy were used teachers witnessed reductions in his/her inattentive behaviors, than with MPH or behavior therapy alone, which confirmed the present studies hypothesis. This might lead future researchers to focus more on drug and therapy treatment as a combination rather than expressing one is better than the other.

\section{References}

Almaric M., Koob G. F. (1993). Functionally selective neurochemical afferents and efferents of the mesocorticolimbic and nigrostriatal dopamine system. Brain Research, 99, 209-226. 
American Academy of Pediatrics. (2001). Subcommittee on attention deficit/hyperactivity disorder and committee on quality improvement. Clinical practice guideline: treatment of the school-aged child with attentiondeficit/hyperactivity disorder. Pediatrics, 108, 10331044.

Barkley, R. A. (1997). ADHD and the nature of self-control. N. Y.: The Guilford Press.

Barkley, R. A. (2003). Attention-deficit/hyperactivity disorder. E.J., Mash \& R.A.,

Barkley (Eds.), Child psychopathology (75-143). New York, N.Y.: The Guilford Press.

Benton, A. (1991). Prefrontal injury and behavior in children. Developmental Neuropsychology, 7, 275-282.

Bidwell, C., McClernon, J., Kollins, S. (2011). Cognitive enhancers for the treatment of ADHD. Pharmacology Biochemistry and Behavior, 99, 262-274. doi: 10.1016/j.pbb.2011.05.002

Bloom, B., Cohen, R., Freeman, G. (2010). Summary health statistics for U.S. Children: national health interview survey. Vital and Health Statistics, 10, 1-78.

Bull, E., Reavill, C., Hagan, J., Overend, P., \& Jones, D. (2000). Evaluation of the spontaneously hypertensive rats as a model of attention deficit hyperactivity disorder: acquisition and performance of the DRL-60s test. Behavioral Brain Research, 109, 27-35.

Cao, J.L., et al. (2010). Mesolimbic dopamine neurons in the brain reward circuit mediate susceptibility to social defeat and antidepressant action. Journal of Neuroscience, 30, 16453-16458. doi: 10.1523/JNEUROSCI.3177-10.2010

Carboni, E., Silvagni, A., Valentini, V., \& Chiara, G. (2003). Effect of amphetamine, cocaine and depolarization by high potassium on extracellular dopamine in the nucleus accumbens shell of SHR rats. An in vivo microdyalisis study. Neuroscience and Biobehavioral Reviews, 27, 653-659.

Casey, B. J., et al. (1997). Implication of right front striatal circuitry in response inhibition and attentiondeficit/hyperactivity disorder. Journal of the American Academy of Child and Adolescent Psychiatry, 36, 374383.

Dupaul, G. J., \& Barkley, R. A.,. (1990). Medication therapy. Barkley, R. A., (Eds.), Attention-deficit hyperactivity disorder: A handbook for diagnosis and treatment (573612). New York, N. Y.: The Guilford Press.

Castellanos, F. X., et al. (1996). Quantitative brain magnetic resonance imaging in attention-deficit hyperactivity disorder. Archives of General Psychiatry,53, 607-616.

Furman, L. (2009). ADHD: what do we really know? Timimi, S., \& Leo, J. (Eds)., rethinking ADHD from brain to culture (21-57). NY: Palgrave Macmillan.

Fuster, J. M. (1997). The prefrontal cortex $\left(3^{\text {rd }}\right.$ ed.). NY: Raven Press.

Giedd, J. N., et al.(1996). Quantitative magnetic resonance imaging of human brain development: Ages 4-18. Cerebral Cortex, 6, 551-560.

Grattan, L. M., \& Eslinger, P. J. (1991). Frontal lobe damage in children and adults: A comparative review. Developmental Neuropsychology, 7, 283-326.

Hinshaw, S. P., Whalen, C. K., \& Henker, B. (1984). Cognitive-behavioral and pharmacological interventions for hyperactive boys: Comparative and combing effects. Journal of Abnormal Child Psychology, 17, 393-408.

Iancu, R., Mohapel, P., Brundin, P., \& Paul, G. (2005). Behavioral characterization of a unilateral 6-OHDAlesion model of Parkinson's disease in mice. Behavioral Brain Research, 162, 1-10.

Klingberg, T., Forssberg, H., \& Westerberg, H. (2002). Training of working memory in children with ADHD. Journal of Clinical Experimental Neuropsychology, 24, 781-791.

Klingberg, T., et al. (2005). Computerized training of working memory in children with ADHD - a randomized, controlled trial. Journal of American Academy Child Adolescence Psychiatry, 44, 177-186.

Koda, K., et al. (2010). Effects of acute and chronic administration of atomoxetine and methylphenidate on extracellular levels of noradrenaline, dopamine, and serotonin in the prefrontal cortex and striatum of mice. Journal of Neurochemistry, 114, 259-270.

Mattes, J.A. (1980). The role of frontal lobe dysfunction in childhood hyperkinesis. Comprehensive Psychiatry, 21, 358-369.

Neill, D.B. (1976). Frontal-striatal control of behavioral inhibition in the rat. Brain research, 105, 89-103.

Nigg, J. T. (2001). Is ADHD an inhibitory disorder? Psychological Bulletin, 125, 571-596.

Paxinos \& Watson Brain Atlas (1986). The rat brain: in stereotaxic coordinates. San Diego, C.A.: Academic Press.

Pelham, W. E., et al. (1988). The combination of behaviour therapy and methylphenidate in the treatment of attention deficit disorders: A therapy outcome study. Journal of Attention Deficit Disorder, 3, 29-48.

Pelham, W. E., et al. (1999). A comparison of morning-only and morning/late afternoon Adderall to morning-only, twice-daily, and three times-daily methylphenidate in children with attention deficit hyperactivity disorder. Pediatrics, 104, 1300-1311.

Peterson, J., Wolf, M., \& White, F. (2003). Impaired DRL 30 performance during amphetamine withdrawal. Behavioral Brain Research, 143, 101-108. doi: 10.1016/S0166-4328(03)00035-4.

Richardson, E., Kupietz, S. S., Winsberg, B. G., Maitinsky, S., \& Mendell, N. (1988).

Effects of methylphenidate dosage in hyperactive reading disabled children: II. Reading achievement. Journal of the American Academy of Child and Adolescent Psychiatry, 27,78-87.

Satterfield, J. H., Satterfield, B., \& Cantwell, D. P. (1981). Three-year multimodality Treatment study of 100 hyperactive boys. Journal of Pediatrics, 98, 650-655.

Sandberg, S. (1996). Hyperactivity disorders of childhood. N. Y.: Cambridge University Press.

Schweitzer, J. B., \& Sulzer-Azaroff, B. (1995). Self-control in boys with attention-deficit hyperactivity disorder: Effects of added stimulation and time. Journal of Child Psychology and Psychiatry, 36, 671-686.

Semrud-Clikeman, M., et al. (2000). Using MRI to examine brain-behavior relationships in males with attention deficit disorder with hyperactivity. Journal of the American Academy of Child and Adolescent Psychiatry, 39, 477-484. 
Shaywitz, S.E., \& Shaywitz, B.A. (1989). Critical issues in attention deficit disorder.

Sagvolden, T. \& Archer, T. (Eds.)., In attention deficit disorder: clinical and basic research (53-69). Hillsdale: Lawrence Erlbaum Associates.

Stuss, D.T., \& Benson, D.F. (1986). The frontal lobes. NY: Raven Press.

Sokolowski, J. D., \& Salamone, J. D. (1994). Effects of dopamine depletions in the medial prefrontal cortex on DRL performance and motor activity in the rat.

Brain Research, 642, 20-28. doi: 10.1016/00068993(94)90901-6

Sonuga-Barke, E., Thompson, M., Abikoff, H., Klein, R., Brotman, L. (2006). Nonpharmacological interventions for preschoolers with ADHD: the case for specialized parent training. Infants \& Young Children, 19, 142-153.

Spencer, T., Biederman, J., \& Wilens, T. (2000). Pharmacotherapy of attention deficit hyperactivity disorder. Child and Adolescent Psychiatric Clinics of North America, 9, 77-99.

Spencer, T. J., et al. (2001). Impact of tic disorders on ADHD outcome across the life cycle: Findings from a large group of adults with and without ADHD. American Journal of Psychiatry, 158, 611-617.

Sprague, R., \& Sleator, E. (1976). Drugs and dosages: Implications for learning disabilities. In R. Knights \& D. Bakker (Eds.), Neuropsychology of learning disorders, 25-36. Baltimore, MD: University Park Press.

Sprague, R., \& Sleator, E. (1977). Methylphenidate in hyperkinetic children: Differences in dose effects on learning and social behavior. Science, 198, 1274-1276.

Taylor, F. B., \& Russo, J. (2001). Comparing guanfacine and detroamphetamine for the treatment of adult attentiondeficit/hyperactivity disorder. Journal Clinical Psychopharmacology, 21, 223-228.

Toplak, E., Connors, L., Shuster, J., Knezevic, B., \& Parks, S. (2008). Review of cognitive, cognitive-behavioral, and neural-based interventions for Attention Deficit/Hyperactivity Disorder (ADHD). Clinical Psychological Review, 28, 801-823.

Volkow, N., Fowler, J., Wang, G., \& Goldstein, R. (2002). Role of Dopamine, the frontal cortex and memory circuits in drug addiction: insight from imaging studies. Neurobiology of Learning and Memory, 78, 610624.

Wanchoo, S.J., Lee, M.J., Swann, A.C., \& Dafny, N. (2010). Bilateral six-hydroxydopamine administration to PFC prevents the expression of behavioral sensitization to methylphenidate. Brain Research, 1312(2), 89-100. doi: 10.1016/j.brainres.2009.11.035

Wilens, T. (2008). Pharmacotherapy of ADHD in adults. CNS Spectr, 13, 11-13.

Zachor, D., Roberts, A., Hodgens, J., Isaacs, J., Merrick, J. (2006). Effects of long-term psychostimulant medication on growth of children with ADHD. Research in Developmental Disabilities, 27, 162-174.

Zametkin, A. J., et al. (1990). Cerebral glucose metabolism in adults with hyperactivity of childhood onset. New England Journal of Medicine, 323, 1361-1366. 